\title{
OPEN Prediction of nodal-line semimetals in two-dimensional black phosphorous films
}

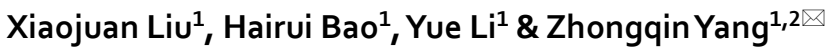

Semimetals are a new kind of quantum materials, in which the conduction and valence bands cross each other near the Fermi level. Based on density-functional theory calculations and symmetry analysis, we propose nodal-line semimetals in layered stacked black phosphorus (BP) films which are designed to have a mirror symmetry lying in the BP layer plane and thus rendering them different from the BP film systems previously studied. A closed nodal-line degenerate band can appear around the Fermi level in the BP films after a biaxial compressive strain is applied. The calculated $Z_{2}$ number of $Z_{2}=-1$ indicates the robustness of the nodal-line semimetals obtained in the BP films, protected by the in-plane mirror symmetry. Intriguingly, with the increase of the film thickness, a smaller biaxial compressive strain is required to produce the nodal-line semimetals, more accessible in experiments. Our results provide a promising route to carrying out the nodal-line semimetals based on various twodimensional materials.

Due to the unique band structures and peculiar properties, semimetals ${ }^{1-15}$ have drawn worldwide attention in recent years, which are generally topologically nontrivial. Topological semimetals (TSMs) can be classified into Dirac $^{1-3} /$ Weyl $^{4,5}$, nodal-line $e^{6-15}$, and nodal-surface ${ }^{15,16}$ semimetals according to the dimensions (zero, one, or two, respectively) of the degenerate energy area formed by the crossing of the conduction and valence bands. The band degeneracy in the semimetals is generally protected by symmetries and is thus robust against external perturbations. The nontrivial topology of the bulk and the exotic surface electronic states in TSMs result in many intriguing quantum transport properties in TSMs, including nearly flat surface states ${ }^{14}$, chiral anomaly ${ }^{17}$, and negative magnetoresistance ${ }^{17,18}$. Currently, plenty of three-dimensional (3D) topological nodal-line semimetals (NLSs) are predicted in theories ${ }^{6-9}$, and some of them have been confirmed in experiments, such as graphene networks ${ }^{6}$ and $\mathrm{Cu}_{3} \mathrm{PdN}^{8}$. Some unique behaviors, including nondispersive Landau energy level ${ }^{14}$, Friedel oscillation ${ }^{19}$, and specific long-range Coulomb interactions ${ }^{20}$, have been proposed in the NLSs. For two-dimensional (2D) NLSs, the exploration is very limited in scope and depth up till now. To the best of our knowledge, some materials with honeycomb-Kagome ${ }^{10}$ or Lieb lattices ${ }^{11}$ were predicted to be $2 \mathrm{D}$ NLSs. Thus, comprehensive theoretical and experimental studies are indispensable for the 2D NLSs.

Black phosphorus (BP) is a layered structure and has potential applications in nanoelectronics owing to its unique behaviors like high carrier mobility, large anisotropy, and negative Poisson's ratio etc ${ }^{21-25}$. 3D BPs, the most stable allotrope of phosphorus, were successfully synthesized in $1914^{26}$ with a layered structure. Each layer of the $\mathrm{BP}$ is a $2 \mathrm{D}$ hexagonal and puckered along the armchair direction. The specially puckered atomic structure of the BP can result in the negative Poisson's ratio in the BP film ${ }^{22}$. Due to the weak interlayer interactions, monolayer or few-layer BPs can be cleaved from the 3D bulk black phosphorus. Monolayer BP flakes with the thickness of $\sim 0.7 \mathrm{~nm}$ were exfoliated by Wang et al. in $2015^{23}$. Based on BP films, field-effect transistors with a high mobility of $\sim 1000 \mathrm{~cm}^{2} / \mathrm{V} / \mathrm{s}$ were fabricated in experiments ${ }^{27}$. Dirac semimetals were reported in experiments in BPs by applying an externally vertical electric field ${ }^{28}$. In theories, $\mathrm{NLSs}^{29}$ were predicted in $3 \mathrm{D}$ BPs by applying hydrostatic pressure etc. For 2D BP films, even though there are numerous previous researches, most of them have focused on digging out the Dirac semimetals ${ }^{30,31}$ and NLSs have not been explored in 2D BP films yet. For future applications, it is meaningful to explore various semimetal behaviors in 2D black phosphorus due to the need for the integration and miniaturization of the devices in nanoelectronics.

In this work, based on density function theory (DFT) calculations and symmetry analysis, we obtain NLSs in 2D layered BP ultrathin films with different thickness for the first time. For a bilayer BP built with a mirror symmetry, an elliptic closed nodal-line degenerate band is found being located around the Fermi level $\left(\mathrm{E}_{\mathrm{F}}\right)$ if a

\footnotetext{
${ }^{1}$ State Key Laboratory of Surface Physics, Key Laboratory of Computational Physical Sciences (MOE) and Department of Physics, Fudan University, Shanghai 200433, China. ${ }^{2}$ Collaborative Innovation Center of Advanced Microstructures, Nanjing 210093, China. ${ }^{\boxplus}$ email: zyang@fudan.edu.cn
} 
(a)
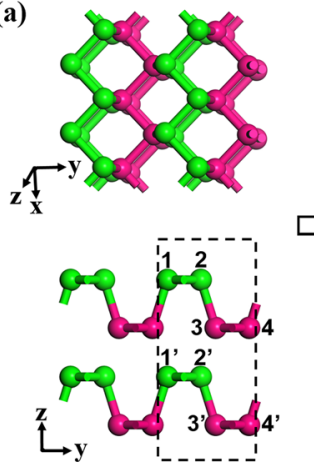
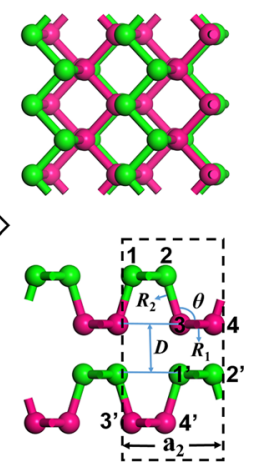

(b)

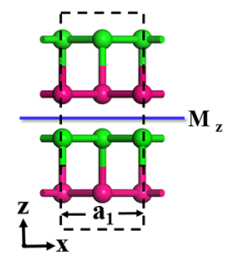

(c)

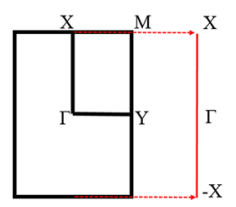

Figure 1. (a) (left panels) Top and side views of the bilayer BP, in which the top layer is stacked vertically on the bottom layer. (right panels) Top and side views of the bilayer BP we study, in which the bottom layer is shifted by $1 / 2$ unit cell along the $\mathrm{x}$ and then $\mathrm{y}$ directions with respect to the top layer. In the built structure, the 1 and 2 atoms in the top layer are above the $3^{\prime}$ and $4^{\prime}$ atoms in the bottom layer, respectively. (b) The other side view (in the xz plane) of the built bilayer BP, owning an $\mathrm{M}_{\mathrm{z}}$ mirror symmetry. The green and magenta colors in (a,b) indicate the top and bottom $\mathrm{P}$ sublattices in the nonplanar monolayer BP. (c) The 2D first Brillouin zone and the projected 1D Brillouin zone of the bilayer BP we build.

\begin{tabular}{|l|l|l|l|l|l|l|}
\hline $\boldsymbol{N}$ & $\boldsymbol{a}_{\mathbf{1}}(\AA)$ & $\boldsymbol{a}_{\mathbf{2}}(\AA)$ & $\boldsymbol{R}_{\mathbf{1}}(\AA)$ & $\boldsymbol{R}_{\mathbf{2}}(\AA)$ & $\boldsymbol{\theta}\left(^{\circ}\right)$ & $\boldsymbol{D}(\AA)$ \\
\hline 2 & 3.298 & 4.628 & 2.220 & 2.260 & 104.175 & 4.411 \\
\hline $2(-5.5 \%)$ & 3.118 & 4.375 & 2.168 & 2.259 & 102.072 & 4.359 \\
\hline 4 & 3.301 & 4.589 & 2.221 & 2.261 & 103.816 & 4.757 \\
\hline 6 & 3.304 & 4.578 & 2.222 & 2.260 & 103.728 & 5.368 \\
\hline
\end{tabular}

Table 1. The optimized lattice constants $\left(a_{1}\right.$ and $\left.a_{2}\right)$, the bond lengths $\left(R_{1}\right.$ and $\left.R_{2}\right)$, the angle between the $R_{1}$ and $R_{2}$ bonds $(\theta)$, and the distance $(D)$ of the layer interval having the mirror symmetry for the multiple layer BP films $(N=2,4,6)$. The second line gives the results of the bilayer BP with a $5.5 \%$ biaxial compressive strain.

certain biaxial compressive strain is applied. The obtained NLS is protected by the mirror symmetry owned in the structure, confirmed by the opposite mirror eigenvalues of \pm 1 for the degenerate bands. Very interestingly, the multilayer BPs, such as the four- or six-layer BPs, are also found to be 2D NLSs, under smaller biaxial compressive strains. The mechanism is analyzed in detail.

\section{Results and discussion}

We focus on $N$-layer BP films $(N=2,4,6)$ to produce the 2D NLS. Since a mirror symmetry possibly leads to nodal-line degenerate bands ${ }^{9,10}$, we built a bilayer BP structure with a mirror symmetry as shown in the right panels of Fig. 1a. The weak interlayer interactions can lead to various stacking orders for the bilayer BPs, which were studied by Zhang et al. ${ }^{32}$ and Dai et al..$^{33}$. The bilayer BP with the same stacking order as the 3D bulk BP has been found owning a quantum spin Hall state after certain controlling schemes are applied ${ }^{32}$. As shown in Fig. 1a, the bilayer BP we studied can be obtained from the bilayer BP, in which the top layer stacks vertically on the bottom layer (in the left panels of Fig. 1a), by shifting the 1 and 2 atoms of the top layer to the positions right above the $3^{\prime}$ and $4^{\prime}$ atoms of the bottom layer, respectively. The designed bilayer BP structure (right panels in Fig. 1a) has an orthorhombic lattice with a layer group 41 (space group 51, Pmma) ${ }^{34}$. The $\mathrm{M}_{\mathrm{z}}$ mirror symmetry owned in the structure is illustrated in Fig. 1b. The structures of four- and six-layer BPs can be similarly built, with the same layer group and $\mathrm{M}_{\mathrm{z}}$ mirror symmetry. The first Brillouin zone (BZ) and the projected one dimensional (1D) BZ of the bilayer BP film built are displayed in Fig. 1c.

The calculated structural parameters of the $N$-layer BP films $(N=2,4,6)$ are listed in Table 1 . The parameters of the bilayer BP with a $5.5 \%$ biaxial compressive strain are also given. For the pristine bilayer BP, the optimized lattice constants are $a_{1}=3.298 \AA, a_{2}=4.628 \AA$. As shown in Table 1, with the increase of the layer thickness $N$, $a_{1}$ increases slightly and $a_{2}$ decreases gradually. $R_{1}$ and $R_{2}$ do not change much with the variation of the layer thickness. The interlayer distance $D$, however, increases obviously with the increase of $N$, showing the weakening of the interlayer interactions between this two BP layers. The applied biaxial compressive strain does not affect the symmetries the system owns. Thus, the crystal with the biaxial strain applied still possesses the mirror symmetry $\left(\mathrm{M}_{\mathrm{z}}\right)$. For the bilayer BP with a $5.5 \%$ compressive strain, the distance $D$ between the two BP monolayers decreases much compared to that of the pristine bilayer BP, ascribed to the obvious decrease of the angle $\theta$ between the $R_{1}$ and $R_{2}$ bonds under the compressive strain. To study the structural stability of the built bilayer BP films, we construct a $4 \times 4 \times 1$ supercell and calculate the frequency dispersion of the bilayer BP along high 

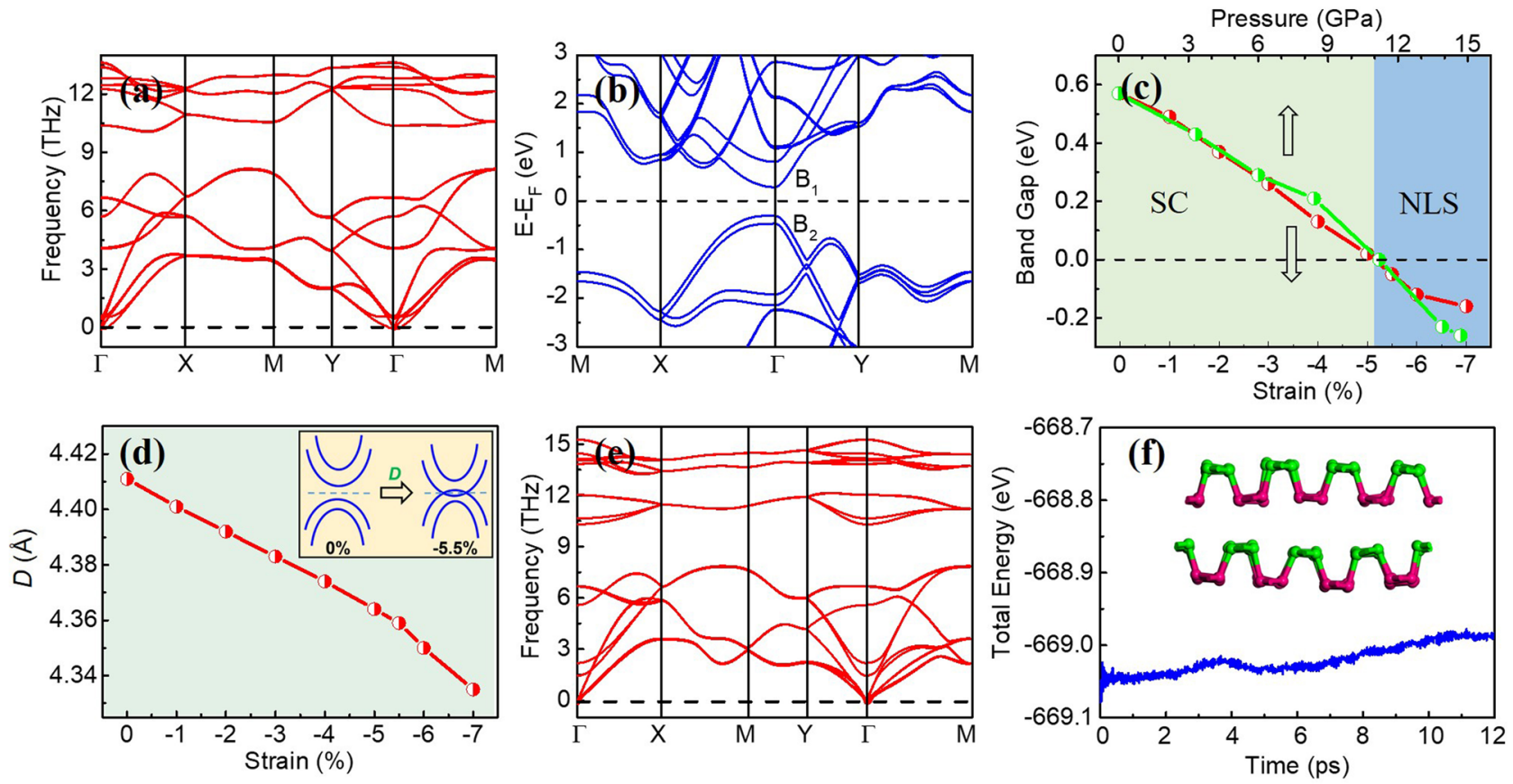

Figure 2. (a) Phonon spectrum of the pristine bilayer BP considered. (b) Band structure of the bilayer BP. The ' $\mathrm{B}_{1}$ ' and ' $\mathrm{B}_{2}$ ' indicate the two bands near to the $\mathrm{E}_{\mathrm{F}}$ (c) Band gap as a function of the biaxial compressive strain (red points) and external pressure (green points) in the bilayer BP. (d) The interlayer distance $(D)$ as a function of the biaxial compressive strain in the bilayer $\mathrm{BP}$. The inset shows the evolution of the bands around the $\mathrm{E}_{\mathrm{F}}$, primarily composed of the $\mathrm{p}_{z}$ orbitals, without and with the biaxial strain. The dash lines in the inset give the $\mathrm{E}_{\mathrm{F} .}$ (e) Phonon spectrum of the bilayer BP under a 5.5\% compressive strain. (f) Total energy fluctuation of the bilayer BP under a 5.5\% compressive strain during the MD simulation at $300 \mathrm{~K}$. The inset in (f) shows the atomic structure of the bilayer $\mathrm{BP}$ at the end of the MD simulation.

symmetry lines in the BZ. As shown in Fig. 2a, no imaginary frequencies appear, indicating that the bilayer BP is dynamically stable at ambient pressure.

The band structure of the pristine bilayer BP is presented in Fig. 2b. The band dispersions along the $\Gamma-\mathrm{X}$ and $\Gamma-Y$ directions are so different, indicating the large anisotropy in the $\mathrm{BP}^{21}$. There is a direct band energy gap of $0.57 \mathrm{eV}$ between the valence band maximum (VBM) and conduction band minimum (CBM) near the $\Gamma$ point. The band gap as a function of the biaxial compressive strain is shown in Fig. 2c. The strain is defined as $\left(l^{\prime}-l\right) \times$ $100 \% / l$, where $l$ ' stands for the lattice constant with a strain applied and $l$ stands for the relaxed lattice constant without the strain. With the increase of the biaxial compressive strain, the band gap decreases gradually. When a $5.2 \%$ compressive strain is applied, the band gap is closed. Note that the band gap is defined as $\mathrm{E}_{\mathrm{B} 1}-\mathrm{E}_{\mathrm{B} 2}$, where $E_{B 1}$ and $E_{B 2}$ are the minimum and the maximum energies of the $B_{1}$ and $B_{2}$ bands, respectively (see Fig. 2b). Thus, the band gap becomes negative when the compressive strain is larger than $5.2 \%$. Hence, the bilayer $\mathrm{BP}$ undergoes a phase transition from a semiconductor (SC) to a metal or semimetal after a compressive strain with a certain magnitude is applied. Besides the strain, applying a pressure is also an effective tuning tactics in experiments. With the extraction method mentioned in Ref. ${ }^{30}$, the band gap as a function of the external pressure is calculated. As displayed in Fig. 2c, when the external pressure reaches $11.2 \mathrm{GPa}$, the band gap becomes zero and the phase transition occurs.

The mechanism of above phase transition is now analyzed. The band gap of the monolayer BP is about 1.51 $\mathrm{eV}^{21}$, while the bilayer BP gives a much smaller band gap $(0.57 \mathrm{eV})$ (Fig. 2b), showing that the interlayer interactions between the two BP monolayers tend to reduce the band gap. For few-layer BP films, the interlayer interactions contain two aspects: the traditional van der Waals (vdW) interaction and the electronic hybridization ${ }^{35}$. The latter comes from the lone electron-pairs of $\mathrm{P}$ atoms due to the three $\mathrm{sp}^{3}$ hybridization orbitals formed on each $\mathrm{P}$ atom. This extra interaction can lead to some exceptional behaviors in the BP film, such as the thicknessdependent vibrational properties etc., not owned by graphene films $\mathrm{s}^{35}$. Thus, the interlayer electronic hybridization and the vdW interaction together influence the electronic states when the interlayer distance $D$ is varied. When a biaxial compressive strain is applied, the $D$ as a function of the strain is displayed in Fig. 2d. The greater the strain strength is, the smaller the distance $D$ is. Thus, the interlayer interactions are strengthened, in favor of closing the band gap. On the other hand, the bands around the $\mathrm{E}_{\mathrm{F}}$ in the bilayer $\mathrm{BP}$ are primarily made up of the $\mathrm{p}_{\mathrm{z}}$ orbitals (see Fig. 3a). Therefore, the band gap of the bilayer BP must be formed due to the bonding and anti-bonding states of the $\mathrm{p}_{\mathrm{z}}$ orbitals, similar to the case in the monolayer $\mathrm{BP}^{24}$. As listed in Table 1 , when a 5.5\% compressive strain is applied, the bond length $R_{2}$ decreases slightly $(0.001 \AA)$, indicating the $\mathrm{p}_{\mathrm{z}}$ bond between the two P atoms (such as the 2 and 3 (or $1^{\prime}$ and $4^{\prime}$ ) atoms in Fig. 1a) in one BP monolayer becomes strong. This trend results in a large band gap between the bonding and anti-bonding states. The $R_{2}$ change is, however, very 

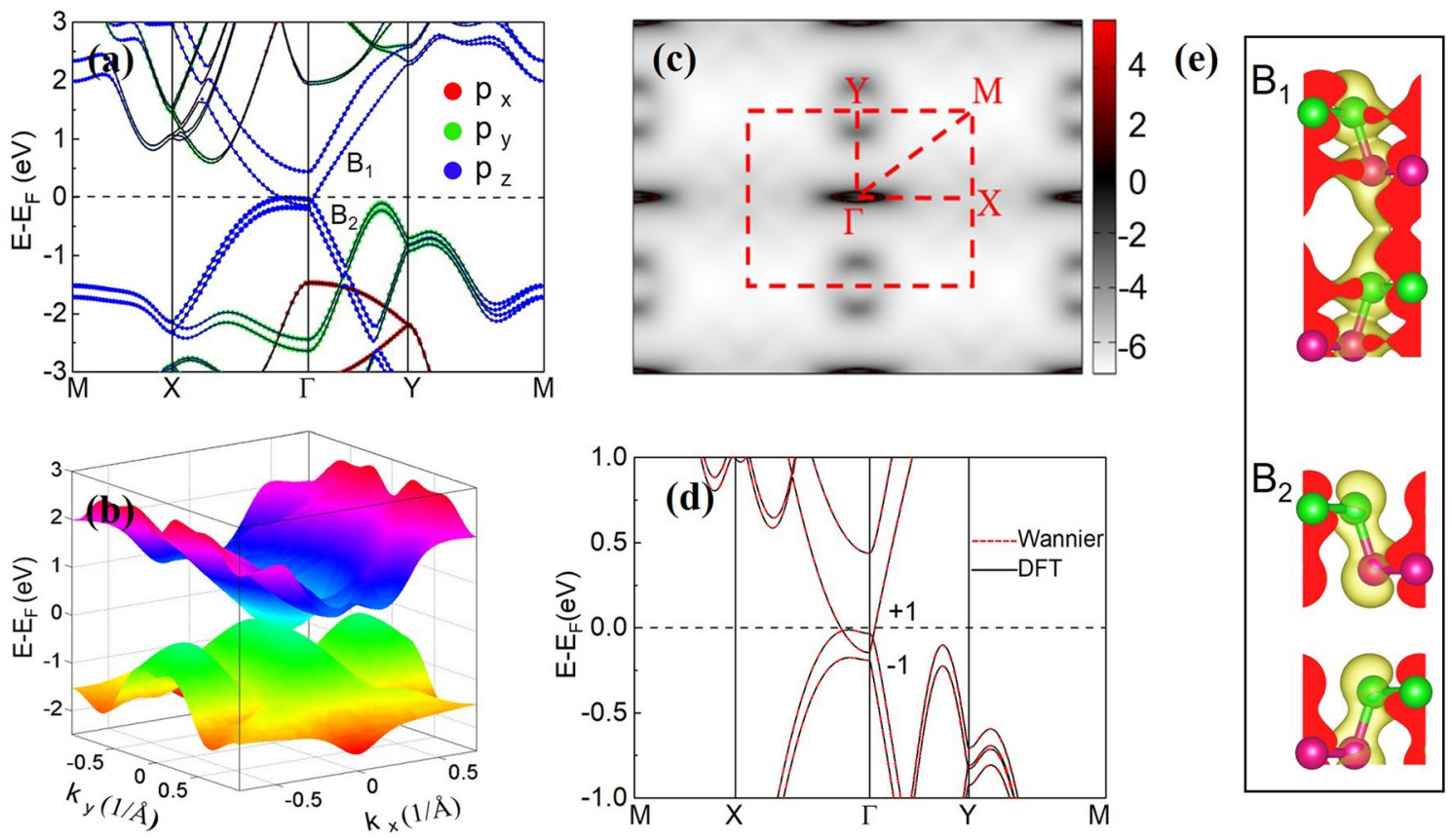

Figure 3. (a) Orbitally resolved band structure for the bilayer BP under a $5.5 \%$ compressive strain. The ' $\mathrm{B}_{1}$ ' and ' $\mathrm{B}_{2}$ ' indicate the two bands closest to the $\mathrm{E}_{\mathrm{F}}(\mathbf{b}) 3 \mathrm{D}$ representation of the band structure shown in (a), indicating a NLS formed around the $\Gamma$ point. (c) Fermi surface in the first BZ of the system. (d) Band structures for the bilayer BP from the DFT (black solid curves) and Wannier interpretation (red dotted curves) calculations. The bands around the $E_{F}$ can be assigned mirror eigenvalues of \pm 1 . (e) The isosurfaces of the charge densities of the $B_{1}$ and $B_{2}$ bands on the yz plane, where the top and bottom $P$ sublattices in the nonplanar monolayer are represented by green and magenta balls, respectively. The isosurface is set to be $0.01 \mathrm{e} / \AA^{3}$.

small with the increase of the compressive strain (Table 1). Hence, the enlarging effect of the band gap from the decrease of $R_{2}$ is negligible, compared to the reduction effect of the band gap from the $D$ decrease in the process. Hence, the main cause of the phase transition from a SC to a metal or semimetal (Fig. 2c) is the increase of the interlayer interactions in the bilayer BP, as illustrated in the inset of Fig. $2 \mathrm{~d}$.

As displayed in Fig. 2c, when the compressive strain is greater than $5.2 \%$, the band gap of the system is closed. To show the bilayer BP entering the NLS state, we take the bilayer BP with a 5.5\% biaxial compressive strain as an example and analyze concretely its electronic states in following. Its structural stability is first explored. Figure $2 \mathrm{e}$ gives the phonon spectrum of the bilayer BP under the 5.5\% compressive strain. Similar to the case without the strain (Fig. 2a), no imaginary frequencies appear in Fig. 2e, inferring the dynamical stability of the structure under the compressive strain. Besides, the thermal stability of the bilayer BP under the $5.5 \%$ compressive strain is also investigated by an ab initio molecular dynamics (MD) simulation. A $4 \times 4 \times 1$ supercell is adopted in the calculations. As illustrated in Fig. 2f, no structural reconstruction happens and the structure remains intact at $300 \mathrm{~K}$ for $12 \mathrm{ps}$, indicating the thermal stability of the bilayer BP under a large compressive strain.

The orbitally resolved band structure for the bilayer BP under the 5.5\% compressive strain is displayed in Fig. 3a. The result clearly reveals that a crossing of the conduction and valence bands, composed of the $\mathrm{p}_{z}$ orbitals, appears around the $\mathrm{E}_{\mathrm{F}}$. This band inversion may lead to interesting band structures. To check whether the bilayer BP forms a NLS near the $\mathrm{E}_{\mathrm{F}}$ under a 5.5\% compressive strain, we plot its 3D band dispersion diagram and the corresponding Fermi surface. As illustrated in Fig. 3b, the highest occupied band and the lowest unoccupied band form a circle of band degenerate intersection near the $\Gamma$ point in the BZ. The Fermi surface plotted in the Fig. $3 \mathrm{c}$ also displays a nodal loop around the $\Gamma$ point. Thus, a NLS is formed in the bilayer BP with a biaxial compressive strain larger than 5.2\%. We check the electronic structures of the bilayer BP film with hybrid Heyd-Scuseria-Emzerhof (HSE06) functional ${ }^{36}$ since the GGA functional may underestimate the band gap. The band gap obtained from the HSE06 is about $1.12 \mathrm{eV}$, larger than that $(0.57 \mathrm{eV})$ from the GGA. And a larger biaxial compressive strain $(>5.2 \%)$ is expected to produce the NLS in the system. Fortunately, as illustrated in Fig. 2c, the phase transition can be induced by applying an external pressure with a not very large magnitude (11.2 GPa). Even if the HSE06 functional is employed, the critical pressure increases to $16.4 \mathrm{GPa}$, still easily carried out in experiments. Besides, as discussed below, the band gap decreases with the increase of the BP thickness. Thus, the NLS may be easily achieved in experiments in the thicker BP films. Due to the weak spin-orbit coupling (SOC) strength of the $\mathrm{P}$ atoms, the SOC effect is ignored in above discussion. If the SOC is considered, all the $2 \mathrm{D}$ crossing points do not exist anymore. Small band gaps appear along the nodal loop, consistent with the trend from the symmetry analysis that the two inverted bands have the same $M_{z}$ eigenvalues instead of different ones. The band gaps opened along the $\Gamma-\mathrm{X}$ and $\Gamma$-Y directions are of 1.5 and $6.6 \mathrm{meV}$, respectively, also reflecting the large anisotropic behaviors of the BP films. Since the band gaps are very small, they are neglected in the following. 
(a)

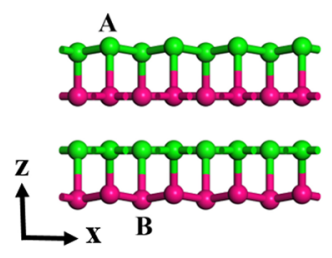

(b)

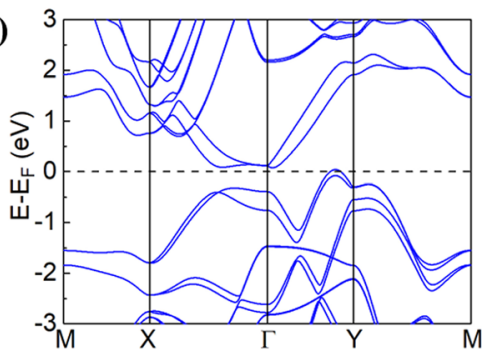

Figure 4. (a) The side view of the bilayer BP with a $5.5 \%$ compressive strain, where the $\mathrm{M}_{\mathrm{z}}$ symmetry is broken by shifting the $\mathrm{A}$ and $\mathrm{B}$ atoms 0.01 and $-0.01 \AA$ along the $\mathrm{z}$ axis, respectively. (b) Band structure of the bilayer BP shown in (a).

To characterize the 2D NLS obtained, we employ mirror eigenvalues of the $M_{z}$ to define the $Z_{2}$ number, as performed in Ref. ${ }^{10}$. The $Z_{2}$ number is calculated through ${ }^{10}$

$$
\mathrm{Z}_{2}=\xi_{\Gamma} \xi_{\mathrm{X}} \xi_{\mathrm{Y}} \xi_{\mathrm{M}}
$$

where $\xi_{a}=\prod_{n=1}^{N_{o c c}} \xi_{a n}$ ( $\xi_{a}$ is the mirror eigenvalue and $\mathrm{N}_{\text {occ }}$ is the number of the occupied states). For the above bilayer BP system, $Z_{2}=-1$ is obtained. Since there is only one nodal line in the BZ (Fig. 3c), we prove that $Z_{2}=(-1)^{N}$, where $N$ is the total number of the nodal lines in a $2 \mathrm{D}$ system, i.e., $\mathrm{N}=1$ and $\mathrm{Z}_{2}=-1$ are obtained for the bilayer BP film. Besides, as illustrated in Fig. 3d, the different mirror eigenvalues of \pm 1 for the two crossing bands also indicate that the degeneracy of the two bands along the nodal loop is protected by the mirror symmetry. Therefore, the formed $2 \mathrm{D}$ NLS in the bilayer $\mathrm{BP}$ is very robust, protected by the mirror symmetry $\mathrm{M}_{\mathrm{z}}$. The isosurfaces of the charge densities of the $B_{1}$ and $B_{2}$ bands (Fig. 3a) on the yz plane are displayed in Fig. $3 e$. A chemical bonding-like character from the $\mathrm{B}_{1}$ band is obviously observed between the two $\mathrm{P}$ monolayers, manifesting the interlayer quasi-covalent (hybridization) interaction, which together with the traditional vdW interaction can tune effectively the band gap under the strain.

To fully understand the origin of the nodal line, we shift the A and B atoms (Fig. 4a) in the bilayer BP with a $5.5 \%$ compressive strain along the $\mathrm{z}$ axis by 0.01 and $-0.01 \AA$, respectively, to artificially break the mirror symmetry $M_{z}$. Note that this bilayer BP without the $\mathrm{M}_{z}$ symmetry is less stable than that of the bilayer BP with the $\mathrm{M}_{z}$ symmetry (Fig. 1b) by $435 \mathrm{meV}$ per unit cell. The corresponding band structure is given in Fig. 4b, from which we find that all the nodes around the $\Gamma$ point are annihilated and the degenerate points are all gapped after the mirror symmetry $\left(\mathrm{M}_{\mathrm{z}}\right)$ of the lattice is broken. An indirect band gap of $64 \mathrm{meV}$ is formed in the band (Fig. 4b). This evidence shows that the nodal-line semimetal obtained in the bilayer BP with a large $(>5.2 \%)$ compressive strain is protected by the mirror symmetry $M_{z}$. Thus, the NLSs may appear in the $2 D$ systems if the $M_{z}$ exists in the structures. If there is a random shift across the film and the mirror symmetry $\mathrm{M}_{\mathrm{z}}$ of the structure is broken, the nodal line does not exist anymore. Due to the relatively weak interlayer interactions of the BP film, a low temperature condition is expected to observe the nodal line behavior in the BP thin film (Fig. 3a).

The electronic states of the $N$-layer BP films with $N=4$ and 6 are also explored. Since the thicker the BP film is, the stronger the interlayer interactions will be, which makes the dispersion of the valence and conduction bands larger, resulting in a smaller band gap. We, thus, expect that the thicker BP films can also possess robust NLSs as the bilayer BP, under maybe smaller biaxial compressive strains. This speculation is confirmed by our DFT results. The $N$-layer BP films ( $N=4$ and 6) also have geometrical structures with a layer group 41 Pmma (space group 51) and $\mathrm{M}_{\mathrm{z}}$ mirror symmetry (Fig. 5a). The dispersion of the low-energy bands of the 4-layer BP film is found to be similar to that of the bilayer. The band gap becomes $0.38 \mathrm{eV}$, smaller than that $(0.57 \mathrm{eV})$ of the bilayer BP. This smaller band gap of the pristine 4-layer BP leads to smaller compressive strains required to close the band gap and trigger the phase transition. Our calculations show that the band gap of the 4-layer BP can be closed with a $3 \%$ biaxial compressive strain. With the further increase of the strain, the band inversion occurs near the $\mathrm{E}_{\mathrm{F}}$ and a NLS is formed in the system. The band structure of the 4-layer BP with a $3.5 \%$ biaxial compressive strain is displayed in Fig. 5b. Similar to the bilayer case, the two crossing bands around the $\mathrm{E}_{\mathrm{F}}$ also have opposite mirror eigenvalues \pm 1 along the high symmetry lines of the $\Gamma-X$ and $\Gamma-Y$ directions and $N=1$ and $\mathrm{Z}_{2}=-1$ are obtained, indicating the robustness of the nodal-line semimetal achieved. For the 6-layer BP, the nodal line is found appearing under a biaxial compressive strain larger than $1.7 \%$ (see Fig. 5c). Thus, a NLS can be more easily acquired in the thicker BP films, favorable for experimental observation.

\section{Conclusion}

Based on first principles calculations and symmetry analysis, we find two-dimensional nodal-line semimetals in the built multiple layer BP films with certain biaxial compressive strains applied. The nodal-line appears around the Fermi level and comes from the inversion of the two $\mathrm{p}_{\mathrm{z}}$ bands with opposite mirror eigenvalues \pm 1 . The $Z_{2}$ $=-1$ shows the robustness of the nodal-line semimetals obtained. The thicker the BP film is, the smaller the compressive strain required to produce the phase transition from an ordinary semiconductor to a nodal-line semimetal will be. Our results provide a route to designing the two-dimensional nodal-line semimetals and promote promising applications of the black phosphorus films in future nanoelectronics. 
(a)
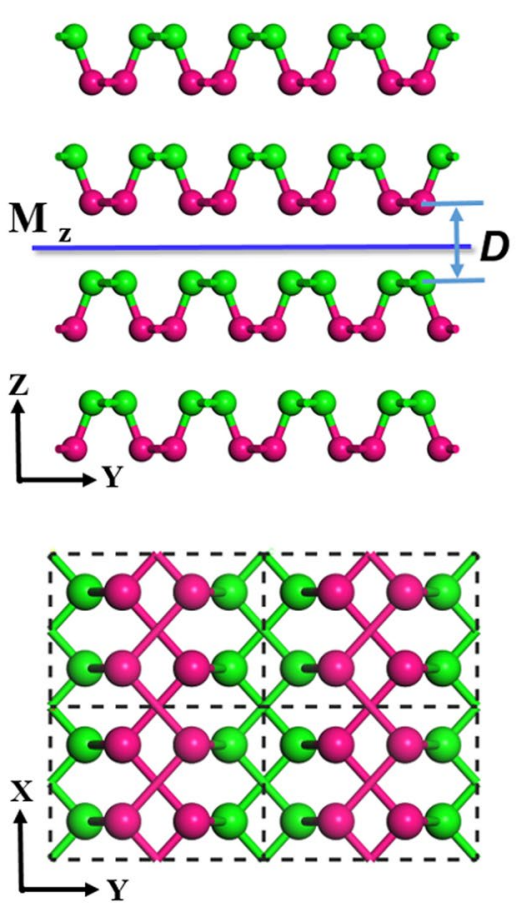

(b)

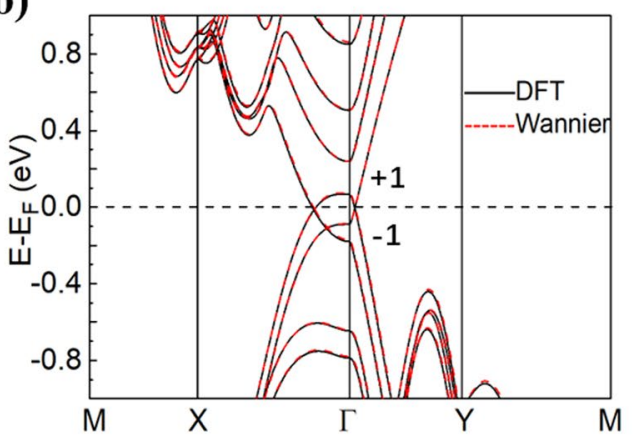

(c)

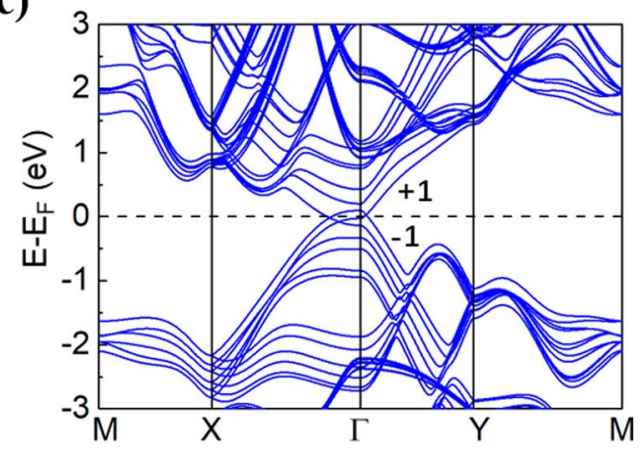

Figure 5. (a) Side and top views of the crystal structure of the 4-layer BP. (b) Band structures of the 4-layer BP under a 3.5\% compressive strain, obtained from DFT (black solid curves) and Wannier interpretation (red dotted curves) calculations. The bands around the $\mathrm{E}_{\mathrm{F}}$ can be assigned with mirror eigenvalues of \pm 1 . (c) Band structure for the 6-layer BP film under a $2.5 \%$ compressive strain. The mirror eigenvalues \pm 1 are also illustrated for the bands around the $\mathrm{E}_{\mathrm{F}}$.

\section{Methods}

Our first-principles calculations are performed based on density functional theory (DFT) using the projector augmented wave method as implement in the Vienna ab initio simulation package (VASP) ${ }^{37}$. The Perdew-Burke-Ernzerhof generalized gradient approximation (GGA-PBE) is adopted for the exchange-correlation functional ${ }^{38}$. The cutoff energy is set as $400 \mathrm{eV}$ for the plane-wave basis and the Brillouin zone is sampled with $\mathrm{k}$ meshes of $14 \times 10 \times 1$ by using Monkhorst-Pack method ${ }^{39}$. Forces on the ions are calculated according to the Hellmann-Feynman theorem. The convergence threshold for the total energy is set to $1 \times 10^{-5} \mathrm{eV}$. The forces on all atoms are optimized to be less than $0.01 \mathrm{eVA}^{-1}$. The phonon state calculations are carried out by using the supercell approach implemented in the PHONOPY code ${ }^{40}$. Molecular dynamics simulations are carried out to determine the thermal stability of the bilayer BP. The maximally localized Wannier functions (MLWFs) are constructed by employing the WANNIER 90 code $^{41}$, from which the Fermi surface is performed by WannierTools package ${ }^{42,43}$.

Received: 15 June 2020; Accepted: 5 November 2020

Published online: 07 December 2020

\section{References}

1. Wang, Z. et al. Dirac semimetal and topological phase transitions in $\mathrm{A}_{3} \mathrm{Bi}(\mathrm{A}=\mathrm{Na}, \mathrm{K}, \mathrm{Rb})$. Phys. Rev. B 85, 195320 (2012).

2. Wang, Z., Weng, H., Wu, Q., Dai, X. \& Fang, Z. Three-dimensional dirac semimetal and quantum transport in Cd $\mathrm{As}_{2}$. Phys. Rev. B 88, 125427 (2013).

3. Liu, Z. K. et al. Discovery of a three-dimensional topological dirac semimetal, $\mathrm{Na}_{3} \mathrm{Bi}$. Science 343, 6173 (2014).

4. Weng, H., Fang, C., Fang, Z., Bernevig, B. A. \& Dai, X. Weyl semimetal phase in noncentrosymmetric transition-metal monophosphides. Phys. Rev. X 5, 011029 (2015).

5. Xu, G., Weng, H., Wang, Z., Dai, X. \& Fang, Z. Chern semimetal and the quantized anomalous hall effect in $\mathrm{HgCr}_{2} \mathrm{Se}_{4}$. Phys. Rev. Lett. 107, 186806 (2011).

6. Weng, H. et al. Topological node-line semimetal in three-dimensional graphene networks. Phys. Rev. B 92, 045108 (2015).

7. Kim, Y., Wieder, B. J., Kane, C. L. \& Rappe, A. M. Dirac line nodes in inversion-symmetric crystals. Phys. Rev. Lett. 115, 036806 (2015).

8. Yu, R., Weng, H., Fang, Z., Dai, X. \& Hu, X. Topological node-line semimetal and dirac semimetal state in antiperovskite Cu ${ }_{3}$ PdN. Phys. Rev. Lett. 115, 036807 (2015).

9. Zhao, J., Yu, R., Weng, H. \& Fang, Z. Topological node-line semimetal in compressed black phosphorus. Phys. Rev. B 94, 195104 (2016).

10. Lu, J. L. et al. Two-dimensional node-line semimetals in a Honeycomb-Kagome lattice. Chin. Phys. Lett. 34, 057302 (2016).

11. Yang, B., Zhang, X. \& Zhao, M. Dirac node lines in two-dimensional Lieb lattices. Nanoscale 10, 1039 (2017).

12. Gao, L. et al. Epitaxial growth of honeycomb monolayer CuSe with dirac nodal line fermions. Adv. Mater. 30, 07055 (2018). 
13. Feng, B. et al. Experimental realization of two-dimensional dirac nodal line fermions in monolayer $\mathrm{Cu}_{2} \mathrm{Si}$. Nat. Commun. 8, 1007 (2016).

14. Rhim, J.-W. \& Kim, Y. B. Landau level quantization and almost flat modes in three-dimensional semimetals with nodal ring spectra. Phys. Rev. B 92, 045126 (2015).

15. Liang, Q.-F., Zhou, J., Yu, R., Wang, Z. \& Weng, H. Node-surface and node-line fermions from nonsymmorphic lattice symmetries. Phys. Rev. B 93, 085427 (2016).

16. Wu, W. et al. Nodal surface semimetals: theory and material realization. Phys. Rev. B 97, 115125 (2018).

17. Huang, X. et al. Observation of the chiral-anomaly-induced negative magnetoresistance in 3D weyl semimetal TaAs. Phys. Rev. X 5, 031023 (2015).

18. Zhang, E. et al. Tunable positive to negative magnetoresistance in atomically thin $\mathrm{WTe}_{2}$. Nano Lett. 17, 04194 (2017).

19. Li, R. et al. Dirac node lines in pure alkali earth metals. Phys. Rev. Lett. 117, 096401 (2016)

20. Huh, Y., Moon, E.-G. \& Kim, Y. B. Long-range Coulomb interaction in nodal-ring semimetals. Phys. Rev. B 93, 035138 (2016).

21. Qiao, J., Kong, X., Hu, Z.-X., Yang, F. \& Ji, W. High-mobility transport anisotropy and linear dichroism in few-layer black phosphorus. Nat. Commun. 5, 4475 (2014).

22. Jiang, J.-W. \& Park, H. S. Negative Poisson's ratio in single-layer black phosphorus. Nat. Commun. 5, 4727 (2014).

23. Wang, X. et al. Highly anisotropic and robust excitons in monolayer black phosphorus. Nat. Nanotechnol. 10, 71 (2015).

24. Popović, Z. S., Kurdestany, J. M. \& Satpathy, S. Electronic structure and anisotropic Rashba spin-orbit coupling in monolayer black phosphorus. Phys. Rev. B 92, 035135 (2015).

25. Yang, F. et al. Quantum hall effect in electron-doped black phosphorus field-effect transistors. Nano Lett. 10, 03267 (2018).

26. Bridgman, P. W. Two new modifications of phosphorus. J. Am. Chem. Soc. 36, 1344 (1914).

27. Li, L. et al. Black phosphorus field-effect transistors. Nat. Nanotechnol. 9, 35 (2014).

28. Kim, J. et al. Observation of tunable band gap and anisotropic dirac semimetal state in black phosphorus. Science 349, 723 (2015).

29. Gong, P.-L. et al. Hydrostatic pressure induced three-dimensional dirac semimetal in black phosphorus. Phys. Rev. B 93, 195434 (2016).

30. Gong, P.-L. et al. Robust and pristine topological dirac semimetal phase in pressured two-dimensional black phosphorous. J. Phys. Chem. C. 121, 20931 (2017).

31. Kim, J. et al. Two-dimensional dirac fermions protected by space-time inversion symmetry in black phosphorus. Phys. Rev. Lett. 119, 226801 (2017).

32. Zhang, T., Lin, J.-H., Yu, Y.-M., Chen, X.-R. \& Liu, W.-M. Stacked bilayer phosphorene: strain-induced quantum spin Hall state and optical measurement. Sci. Rep. 5, 13927 (2015).

33. Dai, J. \& Zeng, X. C. Bilayer phosphorene: effect of stacking order on bandgap and its potential applications in thin-film solar cells. J. Phys. Chem. Lett. 5, 1289 (2014).

34. Wieder, B. J. \& Kane, C. L. Spin-orbit semimetal in the layer group. Phys. Rev B 94, 155108 (2016).

35. Hu, Z.-X., Kong, X., Qiao, J., Normand, B. \& Ji, W. Interlayer electronic hybridization leads to exceptional thickness-dependent vibrational properties in few-layer black phosphorus. Nanoscale 8, 2740 (2016).

36. Heyd, J., Scuseria, G. E. \& Ernzerhof, M. Hybrid functionals based on a screened Coulomb potential. J. Chem. Phys. 118, 8207 (2003).

37. Kresse, G. \& Furthmüller, J. Efficient iterative schemes for ab initio total-energy calculations using a plane-wave basis set. Phys. Rev. B 54, 11169 (1996).

38. Perdew, J. P., Burke, K. \& Ernzerhof, M. Generalized gradient approximation made simple. Phys. Rev. Lett. 77, 3865 (1996).

39. Monkhorst, H. J. \& Pack, J. D. Special points for Brillouin-zone integrations. Phys. Rev. B 16, 1748 (1976).

40. Togo, A. \& Tanaka, I. First principles phonon calculations in material science. Scr. Mater. 108, 1 (2015).

41. Mostofi, A. A. et al. Wannier90: a tool for obtaining maximally-localised Wannier functions. Comput. Phys. Commun. 178, 685 (2008).

42. Sancho, M. P. L., Sancho, J. M. L. \& Rubio, J. Highly convergent schemes for the calculation of bulk and surface Green functions. J. Phys. F Met. Phys. 15, 851 (1985).

43. Wu, Q., Zhang, S., Song, H.-F., Troyer, M. \& Soluyanov, A. A. WannierTools: an open-source software package for novel topological materials. Comput. Phys. Commun. 224, 405 (2017).

\section{Acknowledgements}

This work was supported by National Natural Science Foundation (NNSF) of China under Grant Nos. 11874117 and 11574051 .

\section{Author contributions}

X.L. performed the theoretical calculations with the assistance of H.B. and Y.L.; X.L., H.B., Y.L. and Z.Y. analyzed the results. Z.Y. guided the overall project. X.L. and Z.Y. wrote the manuscript.

\section{Competing interests}

The authors declare no competing interests.

\section{Additional information}

Correspondence and requests for materials should be addressed to Z.Y.

Reprints and permissions information is available at www.nature.com/reprints.

Publisher's note Springer Nature remains neutral with regard to jurisdictional claims in published maps and institutional affiliations. 
(c) (i) Open Access This article is licensed under a Creative Commons Attribution 4.0 International cc) License, which permits use, sharing, adaptation, distribution and reproduction in any medium or format, as long as you give appropriate credit to the original author(s) and the source, provide a link to the Creative Commons licence, and indicate if changes were made. The images or other third party material in this article are included in the article's Creative Commons licence, unless indicated otherwise in a credit line to the material. If material is not included in the article's Creative Commons licence and your intended use is not permitted by statutory regulation or exceeds the permitted use, you will need to obtain permission directly from the copyright holder. To view a copy of this licence, visit http://creativecommons.org/licenses/by/4.0/.

(C) The Author(s) 2020 